\title{
Mobilites Contemporaines, Espace Et Liens Sociaux : Le Nomadisme Reinvente Et Ses Implications En Matiere De Planification Urbaine
}

\author{
Karim Gazzeh, (PhD, Regional Planning) \\ University of Dammam/College of Architecture \& Planning, Saudi Arabia \\ doi: 10.19044/esj.2017.v13n14p195 URL:http://dx.doi.org/10.19044/esj.2017.v13n14p195
}

\begin{abstract}
Since the beginning of the 21st century, the modern society has witnessed an increased mobility as a vector for new lifestyles of the "civilization of mobility".

Tied with the impressive developments in the information and communication technologies, the concept of mobility continues to be a significant challenge that needs to be considered while planning the city or studying the society.

Comparing the contemporary society to the past nomadic communities appears as an approach that would explore and decrypt some socio-spatial phenomena where rapid transformations would be otherwise difficult to analyze using non-comparative methodologies.

This article attempts to revisit some similarities between the contemporary society and the nomadic society in relation to the issue of mobility. The similarities are then analyzed with regard to two key concepts which shape the social pattern on the territories: the spatial reference and the nature of social links.

The article ends with an exploration of proposals on how to take into account the contemporary mobility, henceforth smart, at the level of urban planning policies.
\end{abstract}

Keywords: Contemporary mobility; Nomadism; Urban planning; Social pattern; Social connections

\section{Résumé}

La société moderne connait en ce début du 21ème siècle une montée en puissance des mobilités comme moteur de nouveaux modes de vie marquant résolument l'entrée de l’homme dans la "civilisation de la mobilité”. 
Conjuguée au développement exceptionnel des technologies de l'information et de la communication, la question des mobilités bouscule, structure, déstructure les territoires et pose à ceux qui aménagent ou étudient la ville le défi de la prendre en compte comme mode de questionnement de la société et sur la société.

La comparaison de la société contemporaine aux communautés nomades de jadis émerge très souvent comme une approche qui permettrait d'explorer et décrypter certains phénomènes socio-spatiaux dont les mutations rapides pourraient rendre obsolètes certaines grilles de lecture non comparatives.

Cet écrit propose de revisiter certaines similitudes entre société contemporaine et société nomade en rapport avec la question des mobilités et de les interpréter au regard de deux notions clés qui structurent profondément l'organisation sociale des sociétés sur leurs territoires : le rapport à l'espace et la nature des liens sociaux.

Ensuite, des modalités de prise en compte des mobilités contemporaines, de surcroît connectées, au niveau des politiques de planification urbaine, sont proposées.

Mots clés : Mobilité; Société contemporaine; Nomadisme; Liens sociaux; Planification urbaine

\section{Introduction}

Risquons-nous l'anachronisme, au premier abord, dans un essai de rapprochement des mobilités contemporaines à l'aire nomade de l'histoire humaine ? Cet exercice intellectuel serait-il réducteur des phénomènes au point de se heurter au rejet de la lecture assidue ? ... oui, Probablement.

Mais que risquons-nous à débattre de la question des mobilités qui bouscule, structure et déstructure les territoires ; à donner forme à une réflexion qui affirme d'emblée que rapprochement n'est pas synonyme de "l’histoire se répète" ou que les contextes sont "transposables".

Quelles seraient la nature et l'ampleur des différences qui existent entre la figure contemporaine et la figure nomade et dans quelle mesure objectent-t-elles leur rapprochement?

Mais la tentation d'identifier des liens, des passerelles entre ces deux réalités et leurs modes de vie - dont les séparations ne procèdent pas du seul éloignement temporel - est telle, qu'il serait effectivement intéressant de jauger la portée des similitudes qui autoriseraient la comparaison.

Il s'agit en particulier de révéler la nature des ressemblances/différences et de les interpréter au regard des deux notions qui structurent profondément l'organisation sociale des sociétés sur leurs territoires : le rapport à l'espace et la nature des liens sociaux. 
La question des implications actuelles, en termes de planification de l'espace, est ensuite posée et jaugée afin de savoir si des modalités particulières d'adaptation des grilles de lectures et des modes et méthodes de planification peuvent apporter les réponses adéquates aux transformations induites.

\section{Des formes d'occupation de l'espace fondamentalement différentes}

Après deux révolutions industrielles, une révolution informationnelle et l'ensemble des mutations, vertigineuses par moment, qu'elle a connu, nul doute que la société actuelle est au cœur de l'ère de l'Information et de la Communication où les vitesses et les débits réduisent à néant les distances. Les échanges de toutes sortes sont désormais quasi-instantanés à l'échelle planétaire. Ce processus d’évolution a été déjà amorcé avec l'avènement du train, de la voiture et de l'avion qui ont réduit considérablement les distances métriques. Ceci n’a strictement rien de comparable avec une petite communauté nomade qui arpentait jadis des espaces libres de toute présence humaine significative et contemplait ce que la nature seule a bien voulu dessiner de ses formes et paysages et offrir de ses richesses.

L'Homme, cet être dynamique et inventif porteur de projet pour et/ou contre la nature, a contribué activement à l'apparition puis la confirmation de diverses mutations depuis l'ère lointaine du nomadisme : l'organisation sociale et matérielle des sociétés sur les territoires, les moyens et modes de transport au même titre que les motifs, la périodicité et l'ampleur des mouvements, constituent autant de paramètres qui font de la comparaison un exercice particulièrement difficile tant l'ampleur des différences engloutit toute tentative de rapprochement.

Il est vrai également que les formes d'occupation de l'espace apparaissent fondamentalement différentes entre nomades et "sédentaires" 32 en ce sens que face à un passage relativement "rapide" des nomades, les sédentaires ont toujours marqué l'espace dans une perspective de pérennité. Ceci s'est traduit, progressivement, par des changements socio-culturels et socio-spatiaux et plus particulièrement au niveau des modes de vie.

A ce titre, deux modèles fondamentaux de la mobilité et de ses modes et motifs peuvent être distingués.

Le modèle nomade simple avec une mobilité réduite notamment en termes de fréquence et du caractère “primitif” des facteurs sous-jacents. Et le modèle "sédentaire” plus complexe et plus élaboré.

\footnotetext{
32 Reprenons, le temps de la démonstration, le qualificatif “sédentaire” consacré à l'homme depuis qu'il a été amené à “se fixer”.
} 


\section{Le modèle nomade : Une mobilité réduite pour des motifs simples}

Des mouvements de périodicités longues (saison, année) pour des motifs de recherche de nourriture pour l'homme (chasse, pèches ou cueillette) ou de pâturages pour les animaux, durant la période pastorale. Les nomades scrutent continuellement l'horizon dans l'espoir de nouvelles terres. Des moyens de déplacements primitifs (marche à pied ou animaux domestiqués), des itinéraires qui ne croisent guère des espaces marqués par la présence humaine ou durablement occupés et des formes d'interaction primaire avec les milieux environnants. L'absence d'enracinement spatial est une constante du mode de vie nomade. "Les nomades n'ont bergerie, Qu'il ne suffise à désoler" 33 .

La figure nomade se caractérise par sa simplicité, et probablement par une certaine monotonie, car fondée sur des épisodes répétitifs au gré des saisons et de la disponibilité des richesses : des mouvements cycliques à périodicités égales suivant des itinéraires quasi-identiques. En raison de l'empreinte environnementale mineure qui n'induit pas de modifications profondes des équilibres naturels, les fluctuations de ce modèle n'étaient guère bouleversantes, quoique parfois incertaines.

\section{Le modèle nomade : Une mobilité accrue pour des motifs plus diversifiés}

La société moderne est bien celle où l'émergence puis la confirmation des mobilités comme moteur de nouveaux modes de vie, marquent l'entrée de l'homme dans la "civilisation de la mobilité".

L'étalement urbain, la démocratisation de l'automobile et le développement des moyens de transport par la Mer, la Terre et l'Air, n'en sont en réalité que les facteurs "primaires" de l'essor des mobilités contemporaines. L'imbrication d'autres facteurs, dans un jeu subtil et complexe de causes/conséquences, aboutit à considérer l'ensemble du système d'organisation sociale et matérielle des sociétés sur les territoires et l'apparition désormais perpétuelle de nouveaux modes de vie, de travailler, d'habiter, ..., d'être.

Sur le plan des activités professionnelles, le développement des multinationales tout comme les délocalisations et la nécessité de maintenir les liens avec les centres de décision administrative et de planification logistique, imposent aux entreprises de nouvelles formes d'organisation. Ils induisent également un accroissement considérable des flux de déplacements des personnes au même titre que les marchandises, les fonds et l'information. Ces facteurs constituent aujourd'hui l'un des moteurs clefs des mobilités constatées à périodicité courte, (mensuelle, hebdomadaire, quotidienne).

\footnotetext{
33 Citation de François de MALHERBE, III, 3, extraite de l'article "nomade" du dictionnaire de français Littré.
} 
Dans ce contexte, la diversification des activités conjuguée à l'internationalisation des liens économiques apporte sa contribution à ce mouvement d'ensemble pour en confirmer le caractère de tendance lourde.

Mais il ne s'agit plus uniquement d'activités productives au sens conventionnel. La production culturelle, artistique, sportive ou informationnelle occupe désormais une place grandissante dans cet échiquier économique.

Le droit des citoyens à une information juste, rapide et fiable, l'essor de la transmission en direct et l'impératif d'être au cœur des évènements, induisent au moins deux types de mobilités : une mobilité physique, celle des acteurs de l'information (journalistes et techniciens de l'audiovisuel) et une mobilité symbolique, celle de quelques milliards de téléspectateurs ou auditeurs.

La réalisation d'un film, par exemple, se fait aujourd'hui par séquences dans les quatre coins du monde avec son corollaire en termes de mobilités. Une mobilité physique, celle de l'ensemble des intervenants (acteurs, techniciens, matériel), et une mobilité symbolique, celle de tous ceux qui regardent les films assis sur leurs fauteuils chez eux, ou dans une salle de cinéma à mille lieues des paysages qui défilent devant eux sur l'écran.

Sur un autre registre, le développement impressionnant des activités sportives est un vecteur d'amitié et facteur favorisant la compréhension et les échanges entre les peuples, mais aussi un enjeu économique majeur. Ces activités conjuguent la multitude des disciplines et la diversité des compétitions (nationales, régionales et internationales) à la nécessité d'alterner les lieux d'organisation, pour nous livrer de nouvelles formes de mobilité et compléter un tableau déjà croisé.

Les missions de guerres et de paix, les coopérations internationales diverses, le bénévolat et le travail humanitaire, etc. n’en font, à leur tour, qu'accroître la mobilité.

Par ailleurs, au-delà des motifs professionnels qui peuvent être relativement cernés en rapport notamment avec la division internationale du travail, la globalisation des échanges, les mutations économiques, la redéfinition des structures productives etc., la société moderne est, au même titre, celle des aspirations croissantes aux loisirs rendus possibles grâce à la diminution du temps de travail, l'amélioration des revenus et les performances des infrastructures de transport et des moyens de communication. 
En dehors du contexte malheureux introduit par les séries d'attentats des quinze dernières années ${ }^{34}$, les bouleversements qui ont suivis les révolutions du "Printemps arabe" et les situations d'insécurités et de guerres qui jalonnent douloureusement la chronique, et qui ont affecté les activités touristiques, toutes les statistiques s'accordent à qualifier de prospère l'industrie du tourisme dans le monde. Même si un infléchissement peut être constaté dans certaines régions du monde ces derniers temps, l'élévation du revenu disponible permet depuis quelques décennies à un nombre grandissant de gens de voyager ${ }^{35}$.

On est aujourd'hui de plus en plus nombreux à partir en vacances ou à voyager pour les affaires, pour assister à des réunions, pratiquer un sport, rendre visite à des amis ou parents ou, simplement, pour sortir du quotidien et briser sa routine. Les voyages nous permettent de nous épanouir grâce à de nouvelles expériences, d'éprouver des plaisirs et de nous former, d'apprendre à respecter des cultures étrangères, de nouer des amitiés et de contribuer à la coopération et à la paix internationales.

Plus récemment, les flux migratoires permanents vers les pays de l'Organisation de coopération et de développement en Europe, par exemple, ont représenté 4.3 millions d'entrées en 2014, en augmentation de 6\% par rapport à 2013 (OCDE, 2015). Autant d'âmes qui ont quittés leurs environnements habituels et s'installent à la recherche d'emplois, de nouvelles formes de cohésion sociale, d'intégration et de fonctions d'agrégation.

En 2014, "le nombre total de réfugiés dans le monde s'élevait à 19,5 millions il y a un an. Il a franchi le seuil des 20 millions (20,2 millions) au milieu de 2015 pour la première fois depuis 1992. Les demandes d'asile sont en hausse de 78 pour cent (993 600) par rapport à la même période en 2014. Et le nombre de personnes déplacées dans leur propre pays a bondi d'environ deux millions pour atteindre un total estimé de 34 millions”36.

Différents cas de figures, divers motifs de mobilité, et un dénominateur commun. Les mouvements sont impressionnants. Et on peut

\footnotetext{
${ }^{34}$ Attentats du 11 septembre 2001 aux Etats-Unis, du mois d'octobre 2002 à l'île de Bali (Indonésie), en mars 2004 à Madrid, en juillet 2005 à Londres et à Charm-El-Cheik (Egypte), et plus récemment au mois de janvier et de novembre 2015 à Paris, etc.

${ }^{35}$ Au total, 1,087 milliard de touristes en 2013, soit 52 million de plus que l'année précédente, selon l'Organisation Mondiale du Tourisme. "Malgré les difficultés économiques, les résultats du tourisme international sont bien supérieurs aux attentes", affirme l'OMT (http://www.lemonde.fr/economie/). En 1950, il y avait 20 million de voyageurs internationaux. En 2004 ils étaient 760 million, en progression de $10 \%$ par rapport à l'année 2003, et d'ici à 2020, leur nombre devrait dépasser 1,6 milliard par an, selon la même source.

${ }^{36}$ UNHCR, Agence des Nations Unis pour les réfugiés, Articles d'actualité, 18 décembre 2015: Une hausse sans précédent des déplacements forcés pourrait marquer l'année 2015 [http://www.unhcr.fr/5673c0dfc.html]
} 
multiplier les exemples pour illustrer un paysage et des réalités naturellement et fondamentalement différents de ceux qui prévalaient durant l'aire nomade.

La masse des différences entre les deux figures qui viennent d'être rapidement et probablement fatalement résumées, fait en réalité figure d'assertion tant leur lecture et leur compréhension s’offrent quasiment d'elles-mêmes à l’observateur.

Mais des ressemblances, au niveau de la perception de l'espace et de l'absence d'enracinement socio-spatial, se dévoilent et elles ne semblent pas des moindres !

\section{Des similitudes plus subtiles quant à la perception des espaces traversés}

Ces deux portraits, dont les déplacements, certes différents, opposent, restent à interroger quant à la nature des similitudes qui, vivant au cœur de la première (la figure actuelle), nous rappellent la seconde plus lointaine et que nous connaissons à travers les manuels d'histoire.

Les villes, ces fantastiques créations de l'homme, sièges de ses activités et lieux de ses échanges de toutes natures avec ses semblables (GAZZEH, 2011), sont certes traversées et physiquement présentes dans le schéma contemporain. Mais elles ne se présentent guère au néo-nomade que sous forme de paysages visuels furtifs, à la faveur de la vitesse. Des images qu'on regarde à travers les hublots dans un avion ou dans un train à grande vitesse. Parfois, on s'y arrête, certes. Mais assez souvent pour une correspondance dans la journée ou pour une nuit dans un hôtel parce que le vol de la journée est "surbooké". Certains arrivent, tout de même, à "sortir et visiter” rapidement, dans l'anonymat et "sans laisser de traces”.

Les villes contemporaines prennent des allures de "Transit city"37 dans une planète résolument nomade (BELLANGER et DEVOS, 1997) dans laquelle émerge un paysage subtil traversé par des milliers de néo-nomades qui s’échangent regards et modes de vie et cultivent le goût des lieux de transits (BELLANGER et MARZLOFF, 1996) : aéroports, gares routières ou ferroviaires, marchés, etc.

Dans la société urbaine contemporaine, l'espace paraît fatalement réduit dans sa dimension métrique et dans le même temps symboliquement ignoré voire même dépassé et littéralement vidé de son contenu bâti. Nous traversons "le vide" en quelque sorte malgré la présence physique de la ville et de son contenu social et bâti.

Les néo-nomades, pas nécessairement toujours en situation de mobilité physique, d’ailleurs, vivent aujourd'hui dans les réseaux sociaux,

\footnotetext{
${ }^{37}$ Terme tiré du programme portant le même nom (http://www.transit-city.com) menant des réflexions prospectives sur la ville et les modes de vie et ayant pour ambition de jeter des passerelles entre des domaines aussi divers que l'urbanisme et le marketing, la grande distribution et la sociologie, la promotion immobilière et le transport.
} 
dans les écrans, dans une espèce d'espaces virtuels. Ils communiquent avec des personnes “éloignés” d’eux au sens métrique mais symboliquement plus proches que ceux qui sont présents dans leur environnement physique immédiat. Les adresses classiques laissent leur place aux adresses électroniques. Les achats dans les rayons désertent nos habitudes au profit des commandes en ligne. A un bouquet de fleurs, même promu à une fanaison certaine, mais que l'on peut contempler et sentir, ou une carte postale au format cartonné que l'on peut apprécier au toucher, on préfère désormais les cartes et les bouquets virtuels. Poussant la logique à son extrême, l'espace physique est en passe de disparaitre, ses qualités tangibles sont "gommées".

Les nomades de l’époque lointaine sont-ils si différents à cet égard ?

Ils traversent des espaces physiquement non construits, ou peu occupés, pas plus que les lieux qu'ils investissent et s'y installent, le temps d'une saison, qui ne sont en réalité que de simples lieux de transit. Leurs modes de vie reposant tout naturellement sur une logique de besoins "primitifs" et de mouvements de déplacements périodiques, ils avaient juste des équipements peu encombrants et facilement transportables. Ils "voyagent légers” tout comme le néo-nomade mais pour des raisons différentes.

La symbolique est comparable dans la mesure où les deux figures correspondent à un schéma de mobilité dans lequel seul "le vide” se présente au passant. Parce qu'il se déplace plus vite et plus souvent dans le premier cas, et parce qu’il n’avait guère rien construit n’ayant pas éprouvé le besoin, dans le second.

\section{Des analogies plus profondes quant à l'absence d'enracinement socio- spatial}

La similitude dans le rapport à l'espace introduit à d'autres similitudes dans les rapports sociaux. Il s'agit des transformations qui s'y opèrent résultant de l'essor des mobilités au vu des relations étroites qui existent entre mobilité, transformation des villes et modes de sociabilité (GODARD, 1996).

Les citadins de l'aire contemporaine effectuent plusieurs déménagements successifs au cours de leur vie en fonction de l'évolution de leur statut social. Des trajectoires résidentielles généralement ascendantes et polarisées par l'accession à la propriété, correspondent très souvent à l'amélioration de leur situation socioprofessionnelle (GAZZEH, 2011).

Cette accession à la propriété ne met pas fin, pour autant, à la perpétuité de leur mobilité. D’autres composantes en rapport avec les divers motifs de mobilité restent opérantes (cf. supra). En témoigne la mobilité pour des motifs professionnels : dans un contexte de difficultés d'accès à l'emploi, de précarité ou de licenciements, conjugués aux désirs légitimes d’évolution 
dans le cas d'un emploi stable, plusieurs cas de figures, modulés par la situation de la résidence par rapport à celle du lieu de travail, comme la mobilité pendulaire quotidienne si la personne habite une zone périurbaine, continuent à structurer les modes de vie.

A l'évidence, le repli de l'individu sur son espace personnel ou familial et l'absence de cohésion sociale sont des traits caractéristiques des sociétés contemporaines en quête de fonctions de rassemblement et d'agrégation. La chronique abonde effectivement d'exemples de comportements d'indifférence, de “chacun pour soi” pouvant aller jusqu'à l'absence d'altruisme où “on se détourne de tout” et où on n’éprouve plus le plaisir de la découverte mutuelle (GAZZEH, 2011).

Malgré le caractère réduit du spectre des motifs de déplacements chez les nomades de l'époque lointaine, la perpétuité de la mobilité - qui est une forme primitive de déménagements successifs - n’en demeure pas moins importante même dans le cas classique de mouvements cycliques à périodicités égales suivant des itinéraires identiques et répétitifs. "Le trajet nomade a beau suivre des pistes ou des chemins coutumiers, il n'a pas la fonction du chemin sédentaire (...) il distribue les hommes (ou les bêtes) dans un espace ouvert, indéfini, non communiquant" (DELEUZE et GUATTARI, 1980).

Les communautés nomades sont souvent décrites comme étant solidaires et moins fragmentées, car les relations sociales sont "confinées" dans un cercle familial, tribal ou communautaire restreint. Ce cadre est relativement plus "homogène" et moins complexe que la société contemporaine où la juxtaposition de communautés d'origine et de caractéristiques socioculturelles différentes rend les comportements, les perceptions et les représentations contrastées.

La vie sociale nomade étant moins structurée autour de l'espace qu'autour du temps, les liens sociaux - en tant que conséquence du rapport à un espace défini par "Le territoire" durablement investi et que l'on s'approprie - sont inexistants notamment en ce qui concerne la composante extra-communautaire. Des nuances, toute fois, sont à considérer entre les deux grands types de nomadisme : celui des peuples chasseurs, pêcheurs ou collecteurs et celui des sociétés pastorales.

L'absence d'enracinement socio-spatial ne permet pas non plus le développement des sentiments de citoyenneté et d'appartenance à un espaceterritoire à l'échelle de la cité, de la ville, de la région, ou de la patrie, qui participe positivement à la formation du cadre de vie des individus et garanti l'adhésion de chacun aux œuvres collectives de la communauté.

Cette mobilité, des uns et des autres, peut être interprétée, dans les deux cas, comme un facteur d'isolement dans la mesure où elle ne favorise pas la fixation et l'enracinement. Elle rend les rapports anonymes entre les 
individus, les empêchant de construire une histoire commune et nourrit des formes diverses d'indifférence et de solitude malgré la présence d'une communauté rassemblée autour de l'individu (GAZZEH, 2011).

La symbolique livre ici toute sa signification.

\section{Les implications de l'essor des mobilités en termes de planification de l'espace}

Quelles sont les implications actuelles, en termes de planification de l'espace si ce même espace est, nous venons de le voir, considérablement réduit?

Serait-il pour autant devenu inutile au sens que l'effort de planification que les pouvoirs publics consentent ressemblerait à une perte de temps et de ressources ? Ou cet espace aurait-il muté dans la perception des usagers néo-nomades connectés, et qu'il serait temps de s'adapter tant au niveau des grilles de lectures qu'au niveau des modes et des méthodes de planification ? Serions-nous appelés à repenser l'ensemble du corpus théorique régissant la planification urbaine ?

L’icône des nouvelles générations mobiles et libres cognitivement et politiquement - que Michel Serres appelle "Petite Poucette" (SERRES, 2012) - n'aurait plus besoin, par exemple, des bibliothèques et peut-être pas de l'école telle que nous la connaissons aujourd'hui, en raison des facilitées d'accès au(x) savoir(s) et à l'information rendues possibles grâce à la révolution technologique et informationnelle des dernières décennies et aux vertus du monde à la fois numérisé et librement connecté.

Au-delà du débat, certes passionnant et fructueux, qu'a suscité la mutation civilisationnelle et l'ampleur des transformations de ces dernières décennies que Michel Serres souligne et du nouvel humain qui serait en train de naître (GAUTIER, 2013), la question est de savoir si la planification des services publics, ne serait pas obsolète ou en train de le devenir. Il s'agit des bâtiments éducatifs, culturels, sociaux ou de santé, que les pouvoirs publics en charge de la planification et de la gestion urbaine installent ici et là selon les normes classiques du nombre de populations servies et de la distance métrique de couverture.

Dans le monde actuel où les individus, en perpétuels déplacements, ne sont plus dans une configuration d'enracinement spatial et social, où les distances métriques sont transcendées, où l'enseignement à distance et les services en ligne l'emportent largement sur le présentiel et le face-à-face, a-ton toujours besoins des mêmes normes et outils de planification des services publics. Ces services ne risquent-ils pas de fonctionner en sous régime, exacerbant davantage des coûts de construction et de maintenance déjà exorbitants, et d'être désertés, par endroits, par la nature même des nouveaux modes de vie. 
Risquons-nous de voir le ministère de l'éducation en mal de gérer des écoles ou universités où les enseignants sont "réduits" à concevoir des supports pédagogiques en ligne et des plateformes d'enseignement à distance en contemplant des salles de cours quasiment vides.

Les services sociaux auront-ils toujours besoins d'espaces de sociabilité autres que les réseaux sociaux comme lieux de rencontres et d'échanges et les tablettes numériques comme moyen d'accès.

Les banques auront-ils toujours besoins d'autant d'agences pour une meilleure proximité avec une clientèle qui s'oriente résolument vers des services en ligne et des moyens de paiement utilisant les smartphones.

Les aires de jeux seront-ils aussi fréquentés par des enfants toujours collés à leurs tablettes.

Les musés et les jardins publics seront-ils aussi attractifs pour une population submergée par les offres de visites virtuelles.

En raison des difficultés à estimer correctement les populations à servir, dans le contexte de "non enracinement" spatial, nos calculs savants pour estimer les capacités des réseaux d'alimentation en eau potable risqueraient-ils de causer des pénuries par endroits. Les réseaux d'assainissement pourront ils assurer leur capacité d'auto-curage ici, alors qu'ils seraient surmenés ailleurs.

Force est de constater qu'il est urgent de repenser la sacro-sainte "grille d'équipement publics", a minima. L'espace change, sa mesure et sa lecture devraient, tout naturellement, s'adapter.

Mais comme le réalisme doit l'emporter sur la spéculation et les fantasmes, l'espace physique géographique est bien notre réalité, encore pour longtemps et probablement pour l'éternité. Les mutations sont certes réelles, profondes et vertigineuses. Redéfinition des structures productives et de consommation, travail à distance, nouveaux modes de vie et de sociabilité, nouvelles stratégies résidentielles, ..., nouvelles perceptions du rapport à l'espace.

\section{Conclusion}

Au vu de ses mutations, aux allures de tendances lourdes, et de la nécessité d'y apporter des réponses pertinentes et contemporaines, certaines pistes de réflexion se dégagent avec insistance.

La programmation des services publics urbains, et les politiques de planification urbaine dans leur globalité, devraient indéniablement s'orienter vers plus d'inventivité. Le concept de "Smart city" - que l'on peut traduire par "Ville Connectée" - serait, à juste titre, le point focal de la refonte des méthodes de planification et le "Smart Link" - le lien intelligent - entre ceux qui aménagent ou étudient la ville, d'un côté, et "Petite poucette”, les “Geeks qui débarquent" et tous ceux qui quittent leurs environnements familiers et 
choisissent ou son forcés de s'installer ailleurs, de l'autre. Ils seraient ravis de se réconcilier avec l'espace, l'apprécier pour y vivre leur vie connectée ou tout simplement nouvelle - sans être aliénés.

$\mathrm{Au}$ lieu de chercher à proposer, aux uns, d'arrêter d'être "hyperconnectés” et de réussir leur “détox" digitale, assumant qu’ils sont les esclaves des nouvelles technologies (LEJEALLE, 2015), il serait plus réaliste d'accepter la nouvelle révolution urbaine et de leurs proposer une ville “(...) plus numérique avec son flot de transformations des services urbains et des modes de gouvernance (...)” (BOYER, et WARGNIER, 2015), où bon nombre d'espoirs se concentrent. Philippe Boyer, décrit cette ville avec des termes particulièrement éloquents en écrivant "la ville intelligente ne peut cependant pas se réduire à une vision manichéenne où la technologie serait tout à la fois notre meilleur ami ou notre pire ennemi. Comme toujours, il importe de faire en sorte que le numérique ne soit qu'un outil en faveur d'une ville plus intégrée, plus vivable et plus participative”. Il nous invite à déchiffrer notre condition d'Homo-Urbanus et notre destin d'Homo-Numericus (BOYER, et WARGNIER, 2015).

Au lieu de s’acharner à penser que les migrants constituent la menace du siècle à nos emplois et la turbulence qui envahi notre fleuve tranquille, serions-nous capables d'y voir une richesse supplémentaire et un apport vertueux à une ville qui se transforme pour nous et pour eux, avec nous et avec eux, par ses formes et agencements nouveaux aussi bien au niveau de son bâti que ses tissus urbains, sa voirie et ses espaces publics de sociabilité et de rencontre.

Enfin, il serait plus adéquat de convenir avec Serge Watcher (WATCHER, 2010) que l'ère contemporaine de la mobilité et la société de l'information nous offrent les vertus des vecteurs essentiels de communication et de socialisation avec leurs effets sur les transformations de l'espace urbain.

Il revient aux politiques publiques et aux citoyens, "Citoyens du monde”, d'œuvrer pour que la ville soit écologique tout en étant interactive ou numérique, positive, accueillante et sobre en matière de consommation d'espaces et d'énergies avec des morphologies urbaines et des types d'architecture appropriés pour relever le défi climatique.

Tels sont les défis que la montée de la mobilité, du numérique et de l'écologie soulèvent pour l'urbanisme de demain.

\section{References:}

1. Allemand S., Ascher F., Lévy J. (dir.) (2005). Les sens du mouvement - Modernité et mobilités dans les sociétés urbaines contemporaines, Editions Belin, 336 p. 
2. Bellanger, F., \& Devos, M. (1997). Planète nomade : les modes de vie du passager aérien. Editions de l'Aube., 162 p.

3. Bellanger F., Marzloff B., 1996, Transit - Les temps et lieux de la mobilité, Edition de l'Aube, Paris, 313 p.

4. Bourdin, A. (2004). L'individualisme à l'heure de la mobilité généralisée. In Les sens du mouvement (pp. 91-98), Editions Belin.

5. Boyer Ph., et WARGNIER X. (dir) (2015). Ville connectée = vies transformées - Notre prochaine utopie ? Editions Kawa, 202 p.

6. Deleuze G. et Guattari F. (1980). Traité de nomadologie : la machine de guerre, in Mille Plateaux, pp 434-527, Les éditions de Minuit.

7. Franklin, A. (2008). The tourism ordering. Taking tourism more seriously as a globalizing ordering. Civilisations. Revue internationale d'anthropologie et de sciences humaines, (57), 25-39.

8. Gazzeh K. (2011). Aménagement et risques majeurs, le cas des inondations de novembre 1999 en Languedoc-Roussillon, Edition Universitaires Européennes, Berlin, 397 p.

9. Gautier J. (2013). Petite Poucette : la douteuse fable de Michel Serres, Revue Schole.fr [http://skhole.fr/petite-poucette-la-douteusefable-de-michel-serres], accessed Décembre 2015.

10. Godard F. (1996). Cohésion sociale, dynamique des territoires, Le Courrier du CNRS No 82, Villes, Paris, CNRS, pp. 52-55.

11. Le Breton É. (2004). Mobilité, exclusion et marginalité, in “Les sens du mouvement : modernité et mobilités dans les sociétés urbaines contemporaines” (chapitre 9), Allemand, S. (dir.). Actes du colloque de Cerisy/Institut pour la ville en mouvement, Paris, Belin.

12. Lejealle C. (2015). J'arrête d'être hyperconnecté ! Réussissez votre détox digitale ! 21 jours pour changer, éd. Eyrolles, 224 pages (Format Kindle).

13. Organisation de coopération et de développement économiques OCDE (2015). Perspectives des migrations internationales 2015, OECD Publishing, Paris. DOI : http://dx.doi.org/10.1787/migr_outlook-2015-fr

14. Retaillé D. (1998). Concepts du nomadisme et nomadisme des concepts, in La planète "nomade", les mobilités géographiques d'aujourd'hui”, KNAFOU R. (dir.), Editions Belin, pp. 37-58

15. Raffin, F. (2008). La pensée nomade et Les nouvelles mobilités artistiques contemporaines. In Actes du colloque "Nomadisme, nouveaux médias et nouvelles mobilités artistiques en Europe" (Paris, 21 et 22 février 2008) [http://www. conteners. org/IMG/pdf_La_pensee_nomade. pdf.].

16. Serres M., 2012, Petite Poucette, éditions Le Pommier, 84 p. 
17. Wachter, S. (2010). La ville interactive : l'architecture et l'urbanisme au risque du numérique et de l'écologie. Editions L'Harmattan. 236 p. 